\title{
Das subfasziale venöse Ödem im NMR*
}

\section{Objektivierung des zentralen Korrelates der phlebologi- schen Pathologie?}

\section{T. Stumptner}

Nürnberg

\begin{abstract}
Schlüsselwörter
Beinvenensystem, extravasale Volumenüberlastung, subfasziales venöses Ödem, Faszienspannung, unelastischer Kompressionsverband, Fischer-Technik
\end{abstract}

\section{Zusammenfassung}

Das venöse Blut der Beine wird über ein Venensystem zurück zum Herzen transportiert, wobei die subfaszialen Venen mit einem Anteil von ca. $80 \%$ die Hauptlast tragen. Eine Insuffizienz im System wird über die venöse Hypertonie somit vorwiegend den subfaszialen Raum betreffen. Dieses Drainagedefizit erklärt über die dabei vorliegende extravasale Volumenüberlastung und die daraus folgende vermehrte Faszienspannung die Beschwerden und die klinischen Befunde als Folge der Ödembildung. Das Ödem konnte im MRT dargestellt und seine therapeutische Beeinflussbarkeit mittels unelastischer Kompressionsverbände nach $\mathrm{H}$. Fischer objektiviert werden.

\section{Keywords}

Venous system, extravasal volume overloading, subfascial venous edema, tension of fascia, non-elastic compression bandage, Fischer-Technique

\section{Summary}

For draining the leg of blood exists a venous system. The subfascial veins are the most significant. An insufficiency of the system and the following venous hypertension will be concerned where the mainfunction of the system is situated, in the subfaszial region. By this deficiency of drainage results an extravasal volume overloading (edema) and an increase of the tension of the fascia, what explains the symptoms and the clinical signs. By MRI this edema could be demonstrated and also a therapeutical effect by application of nonelastic compression bandages as designed by Heinrich Fischer.
Korrespondenzadresse

Dr. Thomas Stumptner

Facharzt für Orthopädie, Phlebologie, Chirotherapie

Fürther Str. 244a (Auf AEG)

90429 Nürnberg

Tel. +499112375470

Fax +49 9112375471

E-Mail:info@dr-stumptner.de

www.dr-stumptner.de

* Nach dem Vortrag "Subfascial Edema due to Venous Incompetence demonstrated by MRI" gehalten am 5.5.2017 in Amsterdam beim ICC Annual Meeting „Oedema and Compression”.
Subfascial venous edema by MRI

Phlebologie 2018; 47: 205-209

https://doi.org/10.12687/phleb2404-4-2018

Eingereicht: 16. November 2017

Angenommen: 22. Mai 2018

English version available at:

www.thieme.de/phlebo
Das Beinvenenblut wird über ein Venensystem zurück zum Herzen geleitet. Dabei liegt die Haupttransportleistung mit über $80 \%$ bei den tiefen, subfaszialen Venen (1, 2). Der Blutfluss nach zentral ist ausschließlich durch die Suffizienz der Venenklappen und die effizient arbeitenden sogenannten Venenpumpen gewährleistet, wobei die Funktionstüchtigkeit von Klappen wie von Pumpen von der Funktionstüchtigkeit der Faszie abhängt (3-8). Nur bei Suffizienz dieser drei Strukturen wird alles über die Arterien in das Bein gelangte Blut wieder aus dem Bein hinaus transportiert.

Besteht eine Insuffizienz entweder der Venenklappen oder der Arbeitsfähigkeit der Venenpumpen, resultiert über die Abtransportstörung des Blutes die venöse Volumenüberlastung und in der Folge die venösen Hypertension mit darüber verminderter Rückresorptionsmöglichkeit im Kapillarbereich (9-11).

Umgangssprachlich und damit sofort allgemeinverständlich bedeutet das Reabsorptionsdefizit die unzureichende „Entwässerung", nämlich die "Versumpfung“ des Gewebes. Harvey spricht vom „Ersticken im Überfluss". (12) Es ist klinisch der Zustand der venösen Stauung. Pathophysiologisch wird es als „Ödem“ bezeichnet. Das bedeutet eine extravasale Volumenüberlastung als Folge der gestörten Rückresorptionsmöglichkeit. Diese wird entsprechend der funktionellen Bedeutung der subfaszialen Venen vorwiegend subfaszial relevant sein, wenn auch sie alle Organe des Beines betrifft. Aufgrund der räumlichen Begrenzung des subfaszialen Raumes durch die unelastische Struktur „Faszie“ ist dieser Zustand als vermehrte Faszienspannung gut mit den geklagten typischen Staubeschwerden wie Schweregefühl und Span- 

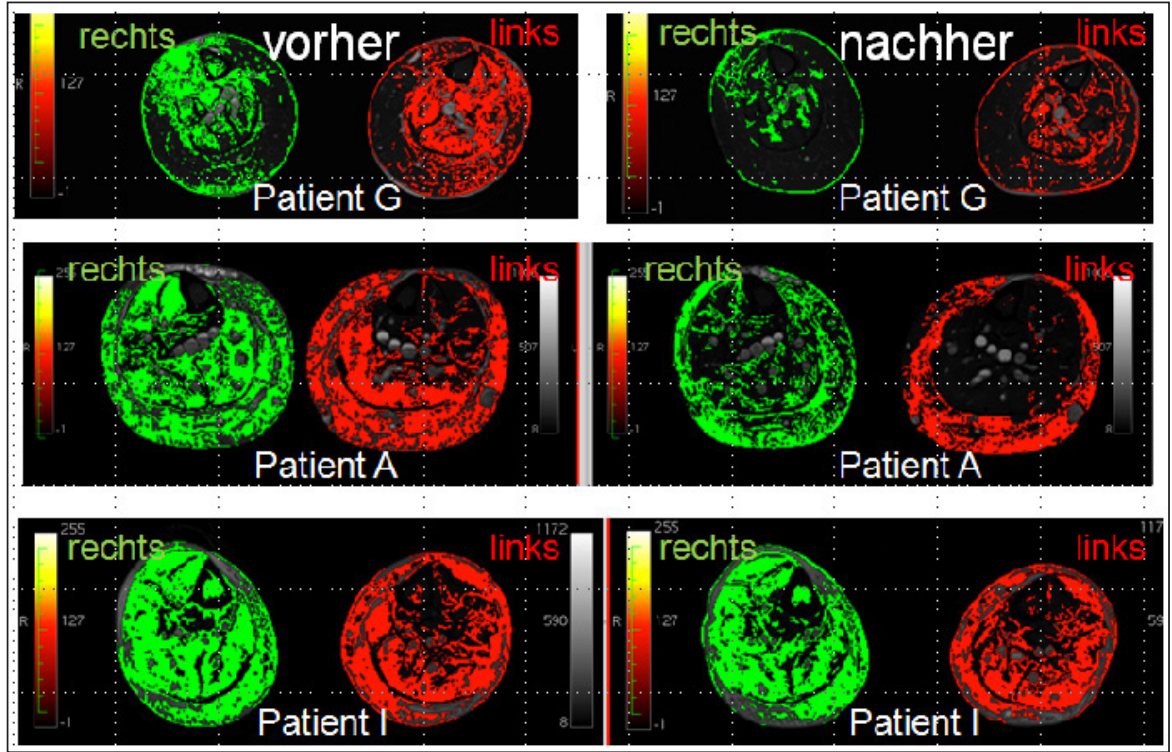

Abb. 1 NMR-Bild in T2-Wichtung der Unterschenkel der Patienten A, G und I vor und nach Behandlung beider Beine, Wassergehalt rechts grün, links rot eingefärbt. Der Wassergehalt ist nach Behandlung reduziert.

nungsgefühl der Beine oder Wadenkrämpfe in Korrelation zu bringen.

Eine Ausprägung des Ödems auch nach epifaszial imponiert gegebenenfalls klinisch als das "dicke Bein“ (13). Vom Patienten wird es eventuell als Schwellneigung empfunden, bei der Inspektion finden sich verstrichene Konturen der Malleolarregion (Bisgaard-Kulisse) und des proximalen Wadenansatzes. Auch eine nicht $\mathrm{zu}$ übersehende Beinumfangsvermehrung kann auftreten.

Die gedankliche Fortführung dieser physiologischen und pathophysiologischen Kaskade, die zur logischen Erklärung dieses subfaszialen Ödemes führt, würde diesen Zustand als Ausgangspunkt weiterer komplizierender Venenerkrankungen erkennen können: einerseits über die vorliegende Venendilatation nach dem Poiseuille'schen Gesetz die Thromboseneigung, andererseits über staubedingte Nutritionsdefizite entzündliche Erscheinungen wie das Stauungsekzem, bis hin zum Gewebetod, dem Ulcus. Die bestehende anhaltende venöse Hypertonie würde die Entwicklung auch einer epifaszialen venösen Insuffizienz erklären.

Diese Gedanken können hier in ihrer Bedeutung allerdings nur genannt werden, zeigen aber die Relevanz des subfaszialen Ödemes (als tastbares klinisches Korrelat), weswegen der Gedanke der vorliegenden Untersuchung zunächst war, das Ödem durch seine Darstellung zu beweisen.

So war es meine Idee, dass dieses Ödem im NMR darstellbar sein müsste, da diese Technik auf der Resonanz der Wasserprotonen basiert. Mit der Technik der Magnetresonanztomographie werden Atomkerne, meist Wasserstoffkerne im Körper resonant angeregt. U.a. liefert der Gehalt an Wasserstoffatomen in Geweben den Bildkontrast der Schnittbilder. Diese setzen sich aus dreidimensionalen Bildpunkten, den sogenannten Voxels zusammen. Es ergeben sich unterschiedliche Graustufen für die verschiedenen Strukturen, die sich zur besseren Darstellung farblich absetzen lassen ( Abb. 1, $\triangleright$ Abb. 4).

Duewell et.al. differenzieren in einer NMR-Untersuchung "geschwollener Beine" Lip-, Lymph- und Phlebödeme, die dabei jeweils deutliche Charakteristika im NMR zeigen (14). Das venöse Ödem - hier allerdings postthrombotisch - stellt sich deutlich abgrenzbar gegenüber den anderen Ursachen dicker Beine betont endofaszial und auch epifaszial dar.

Sehr ähnliche NMR-Bilder zeigen Haarverstad et. al. in einer NMR-Studie des Beinödems (15). Auch hier - zwar postthrombotisch - finden sich Ödeman- sammlungen unter Betonung der subfaszialen Kompartimente.

Die Darstellung des Phlebödemes in beiden Untersuchungen ist identisch mit der Ödemdarstellung in meiner Untersuchung und dokumentiert die Darstellbarkeit dieses Ödemes. Das Lymphödem oder das sogenannte Lipödem imponieren in beiden Untersuchungen im NMR - Bild nach Lage sub- oder epifaszial, nach Struktur und nach Verteilung in den verschiedenen NMR-Wichtungen völlig anders.

Patienten, bei denen neben Beschwerden (Schwere-, Spannungsgefühl der Beine, unruhige Beine, Wadenkrämpfe) der Palpationsbefund (Schwere und Spannung des Gewebes) des subfaszialen Ödemes vorlag, erhielten weiche afferenzstimulierende Schuheinlegesohlen zur aktiven Korrektur einer Fußfehlstatik, sowie die Empfehlung zum Tragen fußfreundlicher Schuhe zur Besserung der Fuß- und Körperhaltung und somit zur Optimierung der faszialen Strukturen im Sinne des „Mädchenfängers" (chinese finger) nach Askar (8) und außerdem die Empfehlung zum mehrfach täglichen zügigen Gehen zur Aktivierung der sogenannten Muskelpumpen. Gleichzeitig wurden sie mit unelastischen Unterschenkelkompressionsverbänden nach Heinrich Fischer (16) behandelt.

\section{Ziel}

Ziel der vorliegenden Untersuchung war die

- Darstellung dieses substanziellen Korrelates dieser pathophysiologischen Sicht, die

- Darstellung des Substrates des Palpationsbefundes der Wade und auch die

- Darstellung des Effektes der Therapie mit dem unelastischen Kompressionsverband am Unterschenkel nach Heinrich Fischer.

Die Hypothese ist, dass durch die Darstellung unterschiedlicher Quantitäten an Wasser im Unterschenkel in den Vergleichsmessungen vor und nach Behandlung die Tatsache des Ödemes und seine therapeutische Beeinflussbarkeit dokumentiert werden kann. 


\section{Material und Methoden}

Fünf Patienten (zwei Männer, drei Frauen, Durchschnittsalter 70,6 Jahre) mit der Diagnose „manifestes subfasziales venöses Ödem" wurden vor und nach Behandlung mit unelastischen Kompressionsverbänden am Unterschenkel im NMR untersucht. Die Diagnose wurde nach Anamnese, Inspektion und Palpation gestellt.

Es wurde eine T2-gewichtete NMR-Untersuchung mit Fettsättigung durchgeführt. T2 -Wichtung bedeutet hohe Signalintensität aller Wasserprotonen. Alle anderen Gewebe zeigen dabei eine niedrige Signalintensität in T2-gewichteten Bildern. Nur Fett würde eine hohe Signalintensität zeigen, weshalb Fettsättigung gewählt wurde. Wenn nur Wasser-Spins eine hohe Signalintensität zeigen, sollte es möglich sein, den Wassergehalt des Gewebes, das Ödem zu messen, indem man alle Voxels oberhalb eines Schwellenwertes zählt.

Im NMR-Bild sind die Signale nicht standardisiert wie im CT. Das NMR-Signal hängt von der Person im Scanner, der Empfängerspule, der Position der Spule und des Patienten zum Magneten und so fort $\mathrm{ab}$. Um Schwellenwerte festzulegen, wurden deshalb übereinstimmende Signale des M. soleus gewählt. Es wurde die mittlere Signalintensität und die Standardabweichung in einer region of interest im M. soleus gemessen und wir definierten die untere Begrenzung des Schwellenwertes als den mittleren Wert aus der ROI abzüglich einer SD und den oberen Schwellenwert als unbegrenzt. Die Voxels innerhalb der Schwellenwerte wurden gezählt und die Summe aller Voxels erklärten wir als den Wassergehalt des Unterschenkels.

(Die radiologische Untersuchung wurde durch Herrn Prof. Dr. R. Janka vom Radiologischen Institut der Universität Erlangen durchgeführt.)

Der Fischerverband (16-19) ist ein Verband mit unelastischen Mullbinden, die präzise unter unterschiedlicher Druckverteilung an den Unterschenkel anmodelliert werden. Sie werden mittels eines Leimes am Bein gehalten. Unelastizität ist das Gegenteil von Elastizität und durch fehlende Dehnbarkeit und damit auch durch eine fehlende Rückstellkraft charakterisiert. In Verbindung mit zügigem Gehen entstehen

Tab. 1 Tabellarische Auflistung der Volumina in $\mathrm{ml}$ bestimmt aus der Summe der Voxels der NMR-Unterschenkelbilder der Patienten A, G, I, und S vor und nach Behandlung und der mit Maßband vor und nach Behandlung bestimmten Werte der maximalen Unterschenkelumfänge.

\begin{tabular}{ll|l|l|l|l}
\hline Name & Zeit & $\begin{array}{l}\text { Volumen rechts } \\
(\mathrm{ml})\end{array}$ & $\begin{array}{l}\text { Volumen links } \\
(\mathrm{ml})\end{array}$ & $\begin{array}{l}\text { Umfang rechts } \\
(\mathrm{cm})\end{array}$ & $\begin{array}{l}\text { Umfang links } \\
(\mathrm{cm})\end{array}$ \\
\hline A & vor & 1355 & 1652 & 40 & 40 \\
\hline A & nach & 797 & 1085 & 36 & 37,5 \\
\hline G & vor & 411 & 835 & 40 & 39 \\
\hline G & nach & 104 & 384 & 38 & 36 \\
\hline I & vor & 664 & 615 & 37,5 & 38 \\
\hline I & nach & 613 & 544 & 34 & 32,5 \\
\hline S & vor & 692 & 579 & 37,5 & 38 \\
\hline S & nach & 136 & (ohne)484 & 34 & (ohne)38 \\
\hline
\end{tabular}

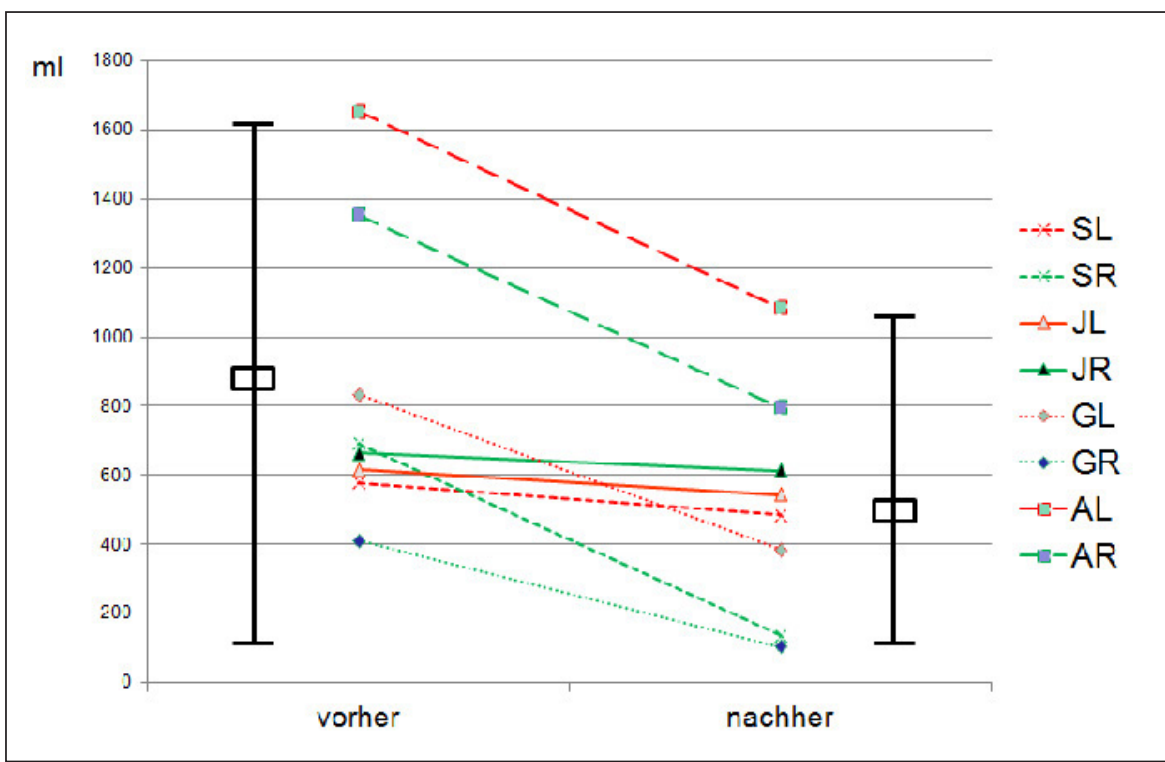

Abb. 2 Flüssigkeitsmenge im Unterschenkel gemessen aus der Summe der Voxels in ml der Patienten $A, G$, I und $S$ vor und nach Behandlung. (Patient $S$ nur rechts behandelt.); $A L=$ Patient $A$ linkes Bein; $A R$ $=$ Patient $\mathrm{A}$ rechtes Bein; $\mathrm{GL}=$ Patient $\mathrm{G}$ linkes Bein; $\mathrm{GR}=$ Patient $\mathrm{G}$ rechtes Bein; IL = Patient I linkes Bein; IR = Patient I rechtes Bein; $S L=$ Patient $S$ linkes Bein; $S R=$ Patient $S$ rechtes Bein

sehr hohe Arbeitsdrucke um die 100 $\mathrm{mmHg}(20,21)$. Diese Therapie wurde bis zur tastbaren Beseitigung des Stauungszustandes durchgeführt.

\section{Ergebnisse}

Mit der NMR-Untersuchung vor und nach Behandlung mittels unelastischer Unterschenkelkompressionsverbände konnte die Reduzierung des Wassergehaltes dargestellt werden. Das entspricht einer Reduktion des Ödemes ( $>$ Abb. 1).
In Zahlen gefasst ergibt sich eine Volumenreduktion aus den Kernspinwerten ( $>$ Tab. 1und $>$ Abb. 2), die mit den am Bein vor und nach Behandlung gemessenen Umfängen ( $>$ Abb. 3) korrespondieren.

Die Relevanz zeigt sich auch deutlich bei einem Patienten, der nur sein rechtes Bein behandeln ließ: die farbliche Darstellung des Wassergehaltes und die Messwerte bleiben am linken unbehandelten Bein gleich, während rechtsseitig sich die Messwerte von Volumen (Voxels) und äußerlich gemessenen Beinumfängen parallel zur 

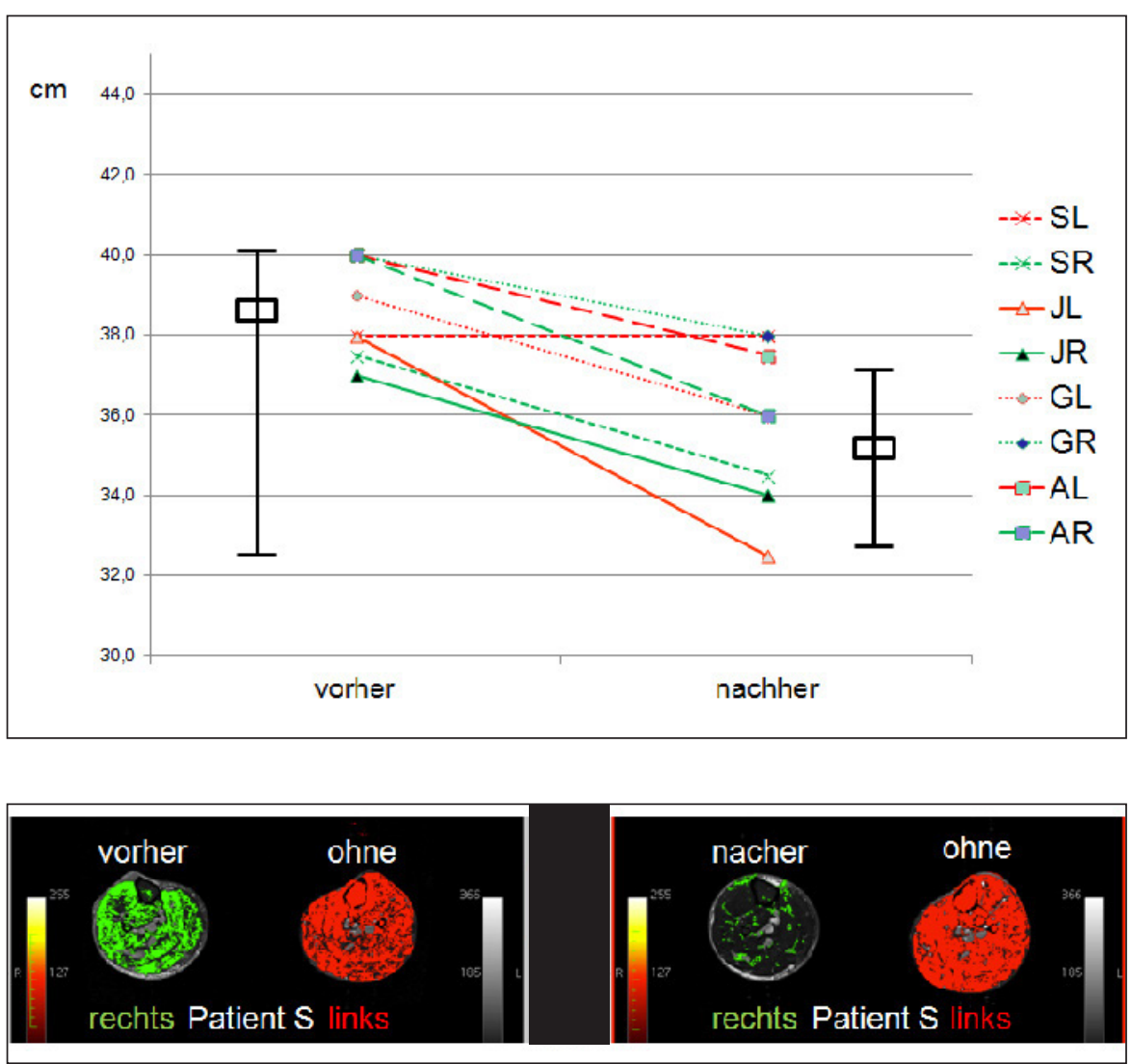

farblichen Darstellung des Flüssigkeitsgehaltes vermindern ( $\triangleright$ Abb. 4$)$.

\section{Schlussfolgerungen}

Entsprechend dem anatomischen und physiologischen Bau des Beinvenensystemes liegt eine sehr hohe funktionelle Bedeutung bei den tiefen Venen im subfaszialen Raum.

Da allerdings der Abtransport des Venenblutes mittels eines Venensystemes geschieht, werden bei einer Insuffizienz des Systemes immer alle Strukturen des Beines betroffen sein.

Logisch erscheint es, dass eine Insuffizienz des Systemes sich vorwiegend im Bereich der Hauptfunktion dieses Systemes, nämlich im subfaszialen Raum manifestiert.

Dieser Gedanke würde mit den Beschwerden der Patienten übereinstimmen, welche sich über die Faszienspannung (22) der flüssigkeitsüberladenen subfaszialen Region durch den subfaszialen Stau erklä- ren. Es würde ebenfalls zum tastbaren Befund der Stauung im Bereich der tiefen Venen der Wade passen.

So vermute ich ein subfasziales Ödem als das pathophysiologische Korrelat von Beinvenenerkrankungen. Ich vermute, dass das Ödem, welches in dieser NMR Untersuchung gezeigt werden konnte, das pathophysiologische Substrat dessen ist, was bereits Browse „The painfull deep-vein Syndrom" (23) nannte und auch das Substrat dessen ist, was Partsch versuchte, als „The painfull deep-vein Syndrom“ und „Pseudothrombophlebitis“ (24) zu differenzieren. Das subfasziale Ödem ist das Korrelat dessen, was Petter (25) als Beschwerden der tiefen Beinveneninsuffizienz beschrieb.

Schon in den frühen 1970er-Jahren zeigten Haid und Partsch in szintigraphischen Untersuchungen den Anstieg des subfaszialen Lymphtransportes bei Patienten mit unelastischer Kompressionstherapie nach Thrombose $(26,27)$ und wiesen damit indirekt das subfasziale Ödem nach. Dies konnte deshalb gezeigt werden, weil der subfasziale Raum durch die bis zur
Abb. 3

Umfangsmaße an der größten Zirkumferenz des Unterschenkels bei den Patienten A, G, I und S vor und nach Behandlung. (Patient $S$ nur rechts behandelt.); $A L=$ Patient $A$ linkes Bein; $A R=P a$ tient $A$ rechtes Bein; $G L=$ Patient $G$ linkes Bein; $\mathrm{GR}=$ Patient $\mathrm{G}$ rechtes Bein; IL = Patient I linkes Bein; IR = Patient I rechtes Bein; SL = Patient S linkes Bein; SR = Patient S rechtes Bein

Abb. 4

NMR-Bild in T2-Wichtung der Unterschenkel des Patienten $S$ vor und nach Behandlung nur des rechten Beines, Wassergehalt rechts grün, links rot eingefärbt. Der Wassergehalt ist am rechten behandelten Bein deutlich reduziert, am linken nicht behandelten Bein unverändert.

Thrombose führende subfasziale Stauung (Rückresorptionsdefizit) bereits „versumpft"war.

\section{Interessenkonflikt}

Nach Angaben des Autors bestehen keine Interessenkonflikte.

\section{Ethische Richtlinien}

Für das Manuskript wurden keine Studien an Menschen oder Tieren durchgeführt.

\section{Literatur}

1. Arnoldi CC. The venous return from the lower leg in health and in chronic venous insufficiency: a synthesis, Acta orth Scand 1964; 35 (Suppl 64): 3-75.

2. Staubesand J. Zur systemischen, funktionellen und praktischen Anatomie der Venen des Beines. In: Schneider W, Walker J (Hrsg). Die chronische Venen-Insuffizienz in Theorie und Praxis, Kompendium der Phlebologie. München: Wolf 1984.

3. Braune W. Die Oberschenkelvene in anatomischer und klinischer Beziehung. Leipzig: Veit 1871. 
4. Schade H, Pich H. Die Pulsationsübertragung von der Arterie auf die Vene und ihre Bedeutung für den Blutkreislauf. Zschr f Kreislauff 1936; 28: 131-172.

5. Schulze W. Über die anatomischen Bedingungen für die Metastasierung bei der Allgemeininfektion, Dtsch Z Chir 1933; 239: 34.

6. Lanz v T, Kressner A, Schwendemann R. Der Einbau der oberflächlichen und der tiefen Venen am Bein, morphologisch und konstruktiv betrachtet. Zeitschr f Anat u Entwicklungsgesch 1936; 108: 695 (Jetzt: Brain strucure and function).

7. Kügelgen $\mathrm{v}$ A. Über den Wandbau der großen Venen. Morph Jb 1951; 91: 447.

8. Askar O, Abou-El-Ainen M. The surgical anatomy of the deep fascia of the human leg. J cardiovasc surg 1963; 4: 114-125.

9. Partsch H. Besserung der venösen Pumpleistung bei chronischer Veneninsuffizienz durch Kompression in Abhängigkeit von Andruck und Material. VASA 1984; 13.

10. Lofferer O, Mostbeck A, Partsch H. Untersuchungen über das Verhalten des Blutvolumens der unteren Extremitäten bei der chronisch - venösen Insuffizienz, Zentralblatt für Phlebologie 1970; 4.

11. Nees S, Juchem G, Weiss DR, Partsch H. Pathogenese und Therapie der Chronischen Venenerkrankung. Phlebologie 2012; 5: 246-257.
12. Harvey W. Exercitatio anatomica de motu cordis et sanguinis in animalibus, Fankfurt 1628. Überstetzt: Ritter von Töply R. Die Bewegung des Herzens und des Blutes. Leipzig 1910.

13. Brunner U, Kappert A, May R, Schoop W, Witzleb E (Hrsg). Das dicke Bein, Grundlagen, Diagnostik, Therapie. Bern 1970.

14. Duewell S, Hagspiel K, Zuber J, Schulthess v G, Bollinger A, Fuchs W. Swollen Lower Extremity: Role of MR Imaging. Radiology 1992; 184(1) 227-231.

15. Haaverstad R, Nilsen G, Myhre H, Säther O, Rinck P. The Use of MRI in the Investigation of Leg Oedema. Eur J Vasc Surg 1992; 6: 124-129.

16. Fischer, Heinrich, Eine neue Therapie der Phlebitis. Medizin Klinik 1910; 30.

17. Fischer, Heinrich. Zur Therapie der Stauungen in den unteren Extremitäten und ihrer Folgen. Münch Med Wschr 1923; 4

18. Haid-Fischer F, Haid H. Venenerkrankungen. Das Wichtigste aus Anatomie, Physiologie, Pathophysiologie und Orthopädie. Stuttgart 1985.

19. Mosti G. Compression treatment in venous insufficiency and arterial disease, Phlebologie 2014; 43 $127-133$

20. Haid H. Ergebnisse fortlaufender Registrierung des Andruckes von Kompressionsverbänden und Gummistrümpfen. In: Molen HR v, Limborgh J v
Boersma W. Progres Cliniques et Therapeutiques dans le Domaine de la Phlebologie. Apeldoorn 1970, 857.

21. Mosti G. Venous ulcer treatment requires inelastic compression. Phlebologie 2018 (1): 7-12.

22. Staubesand J, Li Y. Zum Feinbau der Fascia cruris mit besonderer Berücksichtigung epi- und intrafaszialer Nerven, Manuella Med 1996; 34: 196-200.

23. Browse NL. The painfull deep-vein symdrom. Lancet 1970, 1251-1253.

24. Partsch H „Painfull deep-vein“ Syndrom - Pseudothrombophlebitis in: Brunner, U., Der Unterschenkel, Bern 1988

25. Petter O. Beinbeschwerden bei Leitveneninsuffizienz. In: Schöpf E, Staubesand J (Hrsg). Das schmerzende Bein. Bonn 1992.

26. Haid H, Lofferer O, Mostbeck A, Partsch H. Die Lymphkinetik beim Postthrombotischen Syndrom unter Kompressionsverbänden. Med Klin 1968; 63: 754-757.

27. Lofferer O, Mostbeck A, Partsch H., Nuklearmedizinische Diagnostik von Lymphtransportstörungen der unteren Extremität, VASA 1972; 1: 94-102. 